\title{
The Design of Harmonic Simulator based on Hartley Transform
}

\author{
${ }^{* 1}$ Fahri Vatansever and ${ }^{1}$ Nedim Aktan Yalcin \\ ${ }^{1}$ Faculty of Engineering, Department of Electrical-Electronics Engineering, Uludag University, Turkey
}

\begin{abstract}
Increase in the number of nonlinear loads and devices in energy and power systems have raised harmonic distortion on voltage and current signals. Harmonics are one of the primary factors in determining power quality. Therefore, measurement and control of harmonics are crucial. Many methods have developed for harmonic analysis. In this study, a simulator which uses Hartley transform for determining harmonics is designed. Harmonic distributions and parameters of loaded/defined signals can be calculated accurately and effectively and obtained results can be displayed numerically and graphically with developed systems which have user friendly interface.
\end{abstract}

Key words: Hartley transform, harmonic analysis, simulator.

\section{Introduction}

Harmonics have crucial role in energy and power systems. Measuring and controlling harmonics are basic elements for power quality. For his purpose, many methods have been developed such as Fourier transform, Prony method, wavelet transform, Hilbert-Huang transform, Park transform, chirp-z transform, Kalman filters, artificial neural networks (ANN), phase locked loop (PLL), enhanced phase locked loop (EPLL), adaptive linear element (ADALINE), autoregressive moving average (ARMA), evolutionary algorithms, least squares approach, support vector machines (SVM), Pisarenco harmonic decomposition, (PHD), multiple signal classification (MUSIC), estimation signal parameters via rotational invariance techniques (ESPRIT), and adaptive filters. Types of harmonics and basic methods which are used for estimation, calculation and measurement of harmonics can be classified as shown in Table 1 and Figure 1 [1-2].

Table 1. Harmonic types

\begin{tabular}{|c|c|c|}
\hline Harmonic & Frequency & Description \\
\hline$\overline{D C}$ & $f=0$ & \multirow{4}{*}{$\begin{array}{c}f: \text { Frequency } \\
f_{0}: \text { Main frequency } \\
n: \text { Positive integer }\end{array}$} \\
\hline Harmonic & $f=n \cdot f_{0}$ & \\
\hline Inter-harmonic & $f \neq n . f_{0}$ & \\
\hline Sub-harmonic & $0<f<f_{0}$ & \\
\hline
\end{tabular}

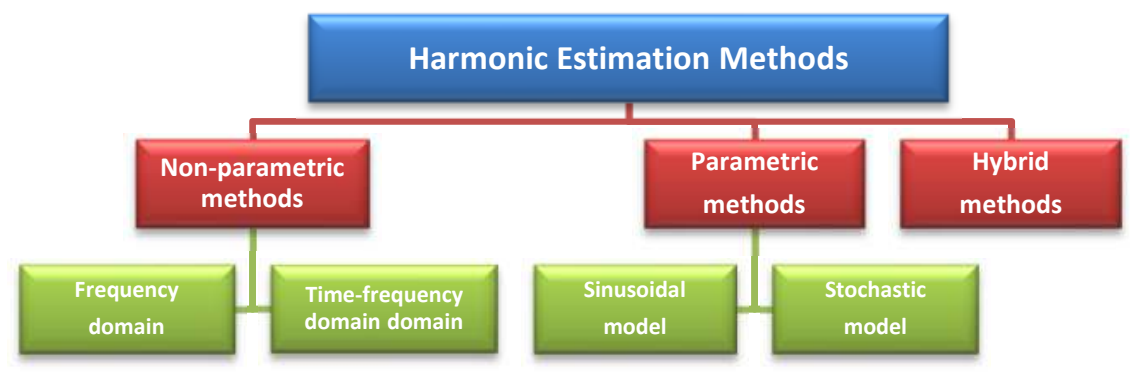

Figure 1. Basic grouping of harmonic estimation methods

In this study, calculation of harmonics was realized with Hartley transform. Simulator which has

*Corresponding author: Address: Faculty of Engineering, Department of Electrical-Electronics Engineering, Uludag University, 16059, Bursa, TURKEY. E-mail address: fahriv@uludag.edu.tr, Phone: +902242940905 
user friendly interface and educational functionalities was designed then harmonic analyzes were carried out with this simulator. As a result of realizations, it was observed that Hartley transform can be easily used in this area.

\section{Hartley Transform}

Hartley transform is an integral transform which maps a real-valued time domain function to realvalued frequency domain function. In this transform, Equation 1 is used for kernel function.

$$
\operatorname{Cas}(\omega t)=\operatorname{Cos}(\omega t)+\operatorname{Sin}(\omega t)
$$

Continuous and discrete Hartley transform of any $x(t)$ function are given in Table 2-3[3-4]. From Table 2, it is easily noticed that Fourier transform of any $x(t)$ function is difference of even part and " $j$ " times odd part of Hartley transformation of this function.

Table 2. Continuous Hartley transform

\begin{tabular}{|c|c|c|c|}
\hline \multicolumn{2}{|c|}{ Continuous Hartley Transform } & $\omega=2 \pi f \quad, \quad H(f)=\sqrt{2 \pi} H(\omega)$ \\
\hline \multicolumn{2}{|c|}{ Angular (rad/s) frequency $[\omega]$} & & Frequency $\left(\mathbf{s}^{-1}\right)[f]$ \\
\hline & $H(\omega)=\frac{1}{\sqrt{2 \pi}} \int_{-\infty}^{+\infty} x(t) \operatorname{Cas}(\omega t) d t$ & & \\
& $x(t)=\frac{1}{\sqrt{2 \pi}} \int_{-\infty}^{+\infty} H(\omega) \operatorname{Cas}(\omega t) d t$ & $\int_{-\infty}^{+\infty} x(t) \operatorname{Cas}(2 \pi f t) d t$ \\
\hline $\begin{array}{c}\text { Even } \\
\text { part }\end{array}$ & $H_{e}(f)=\frac{H(f)+H(-f)}{2}=\int_{-\infty}^{+\infty} x(t) \operatorname{Cos}(2 \pi f t) d t$ & $\begin{array}{l}\text { Odd } \\
\text { part }\end{array}$ & $\int_{-\infty}^{+\infty} H(f) \operatorname{Cas}(2 \pi f t) d t$ \\
\hline
\end{tabular}

Table 3. Discrete Hartley transform

\begin{tabular}{|c|c|c|c|}
\hline \multicolumn{3}{|c|}{ Discrete Hartley Transform } \\
\hline Type & Definition & Type & Definition \\
\hline Type-I & $X[n]=\sum_{k=0}^{N-1} x[k] \operatorname{Cas}\left(\frac{2 \pi k n}{N}\right)$ & $X[n]=\sum_{k=0}^{N-1} x[k] \operatorname{Cas}\left(\frac{\pi k(2 n+1)}{N}\right)$ \\
\hline Type-II & $X[n]=\sum_{k=0}^{N-1} x[k] \operatorname{Cas}\left(\frac{\pi(2 k+1) n}{N}\right)$ & Type-IV & $X[n]=\sum_{k=0}^{N-1} x[k] \operatorname{Cas}\left(\frac{\pi(2 k+1)(2 n+1)}{2 N}\right)$ \\
\hline
\end{tabular}

$$
\begin{gathered}
X(f)=F\{x(t)\}=\int_{-\infty}^{+\infty} x(t) e^{-j 2 \pi f t} d t=\underbrace{\int_{-\infty}^{+\infty} x(t) \operatorname{Cos}(2 \pi f t) d t}_{H_{e}(f)}-j \underbrace{\int_{-\infty}^{+\infty} x(t) \operatorname{Sin}(2 \pi f t) d t}_{H_{o}(f)} \\
=H_{e}(f)-j H_{o}(f)=\frac{H(f)+H(-f)}{2}-j \frac{H(f)-H(-f)}{2}=\frac{1}{2} e^{-j \frac{\pi}{4}} H(f)+\frac{1}{2} e^{j \frac{\pi}{4}} H(-f) \\
\operatorname{Re}\{X(f)\}=H_{e}(f)=H_{e}(-f) \quad, \quad \operatorname{Im}\{X(f)\}=-H_{o}(f)=-H_{o}(-f)
\end{gathered}
$$

Similarly, Hartley transform of any $x(t)$ function is difference of imaginary and real parts of Fourier transform of this function. 


$$
H(f)=H_{e}(f)+H_{o}(f)=\operatorname{Re}\{X(f)\}-\operatorname{Im}\{X(f)\}=\frac{1+j}{2} X(f)+\frac{1-j}{2} X(-f)
$$

\section{Designed Simulator and Its Applications}

Harmonic analysis of signals which are uploaded from file or created in program are realized with simulator which is designed using MATLAB[5] and implements Hartley transform (Table 3 ) as an alternative to conventional approaches such as fast Fourier transform (FFT). Distributions of harmonics are showed both numerically and graphically as a result of relative analyzes. Besides, if $X_{i}$ represents any amplitude elements of harmonics in $x(t)$ signals, effective value, total harmonic distortion, form factor and crest factor of $x(t)$ can be calculated as in Table $4[6-8]$.

Tablo 4. Harmonic parameters which is calculated in simulator

\begin{tabular}{|c|c|c|c|}
\hline Effective Value (RMS) & $\begin{array}{c}\text { Total Harmonic Distortion } \\
\text { (THD) }\end{array}$ & Form Factor (FF) & Crest Factor (CF) \\
\hline$X_{R M S}=\sqrt{\frac{1}{T} \int_{T} x(t)^{2} d t} \cong \sqrt{\sum_{i=1}^{n} X_{i}^{2}}$ & $X_{R M S}=\frac{\sqrt{\sum_{i=2}^{n} X_{i}^{2}}}{X_{1_{R M S}}}$ & $X_{R M S}=\frac{X_{R M S}}{X_{1_{R M S}}}$ & $X_{R M S}=\frac{x_{\max }}{X_{R M S}}$ \\
\hline
\end{tabular}

Screenshot of first analysis realized with $x(t)$ signal which is given Equation 4 and has $f=50 \mathrm{~Hz}$ frequency value is presented in Figure 2.

$$
x(t)=220 \operatorname{Sin}(\omega t)+33 \operatorname{Sin}(3 \omega t)+22 \operatorname{Sin}(7 \omega t)+11 \operatorname{Sin}(15 \omega t)
$$

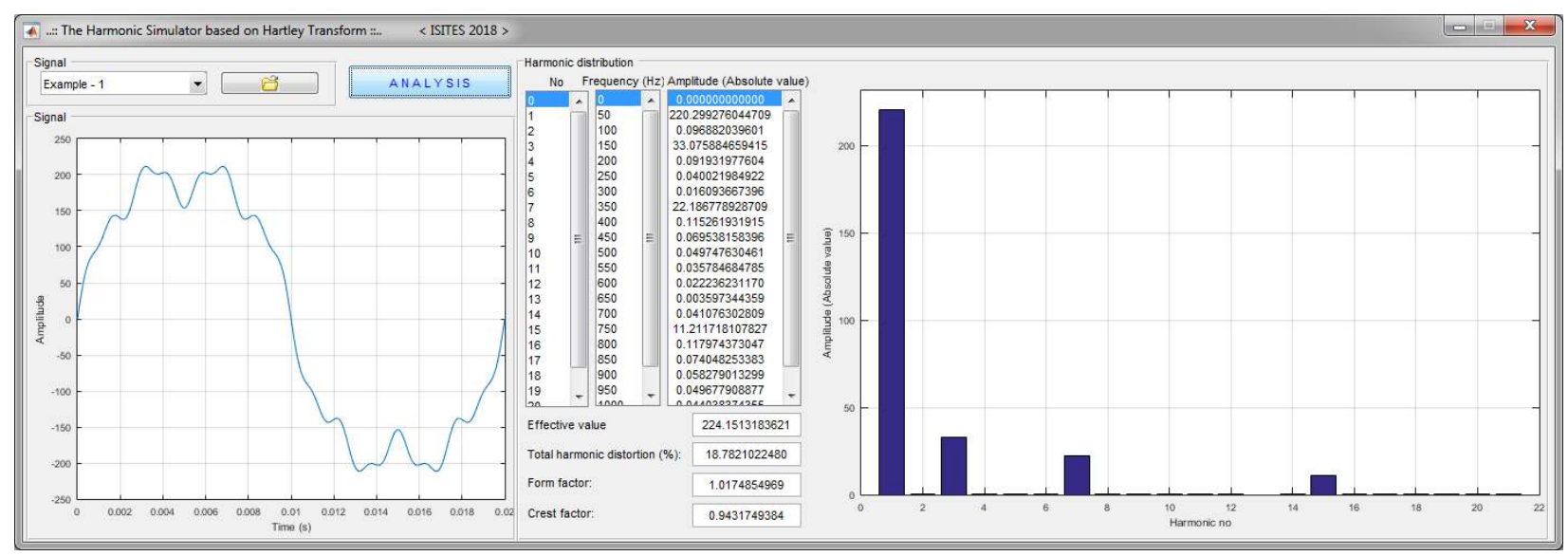

Figure 2. Screenshot of first simulation

Screenshot of second analysis realized with $x(t)$ signal which is sampled and uploaded from file (Eq. 5, $f=50 \mathrm{~Hz}$ ) is presented in Figure 3.

$$
x(t)=0.5+1.0 \operatorname{Sin}(\omega t)+0.3 \operatorname{Sin}(5 \omega t)+0.2 \operatorname{Sin}(11 \omega t)+0.1 \operatorname{Sin}(19 \omega t)
$$


As result of these simulations, using Hartley transform, harmonic distribution can be obtained as high accuracy as Fourier transform. Thus, harmonic analyzes of signals can be calculated effectively and precisely by using fast Hartley transform algorithms.

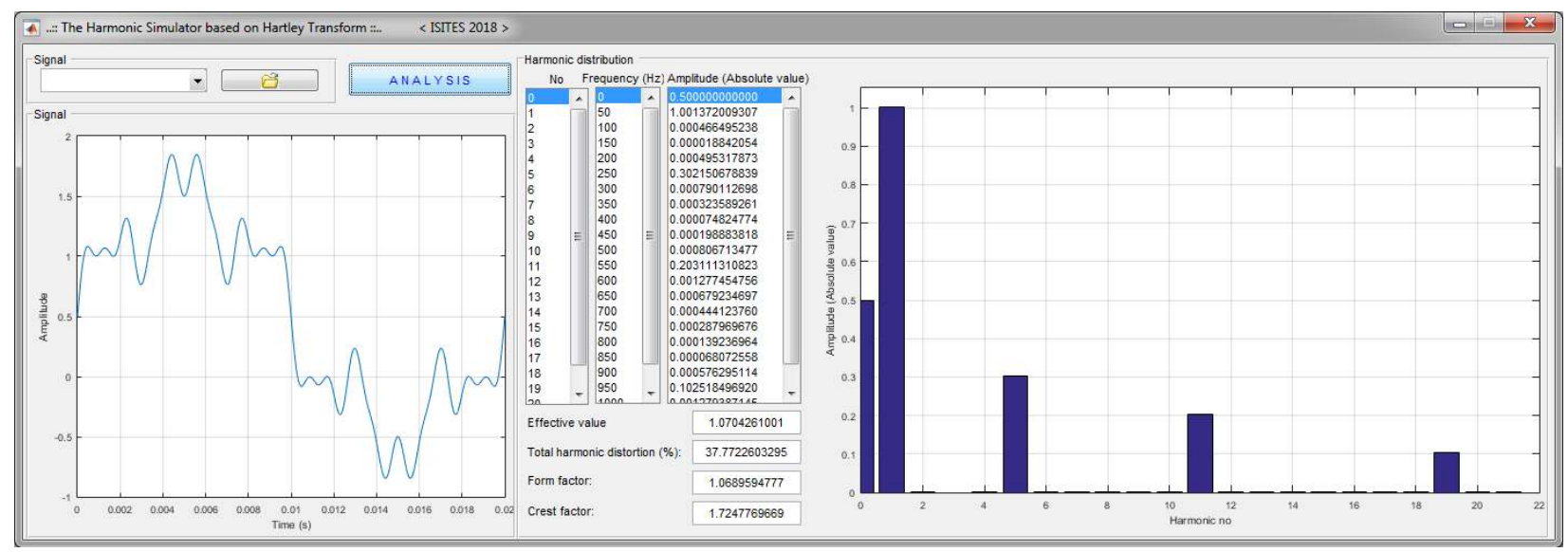

Figure 3. Screenshot of second simulation

\section{Conclusions}

In this work, graphical interface program based on Hartley transform is designed for harmonic analysis. Harmonic analysis and related parameters of signals can be calculated with designed program which implements Hartley transform and can also be used for educational purposes thanks to its interactive, user friendly interface. In realized study, it is clear that harmonic distribution of signals can be easily obtained using Hartley transform and this transform can be used as an alternative method to conventional methods such as Fourier transform.

\section{References}

[1] Jain SK, Singh SN. Harmonics estimation in emerging power system: Key issues and challenges. Electric Power Systems Research 2011; 81: 1754-1766.

[2] Singh GK. Power system harmonics research: a survey. European Transactions on Electrical Power 2009; 19: 151-172.

[3] Poularikas AD (Ed.). Transforms and applications handbook. 3rd ed. USA: CRC Press; 2010.

[4] Bi G, Zeng Y. Transforms and fast algorithms for signal analysis and representations. USA: Springer Science+Business Media, LLC; 2004.

[5] MATLAB, The MathWorks Inc., https://www.mathworks.com/

[6] Tolbert LM, Hollis HD, Hale PS. Survey of harmonic measurements in electrical distribution systems. IEEE IAS Annual Meeting, San Diego, CA, 6-10 October 1996, pp. 2333-2339.

[7] Sankaran C. Power quality. CRC Press LLC; 2002.

[8] Halpin SM. Power quality: The electric power engineering handbook. CRC Press LLC; 2001. 